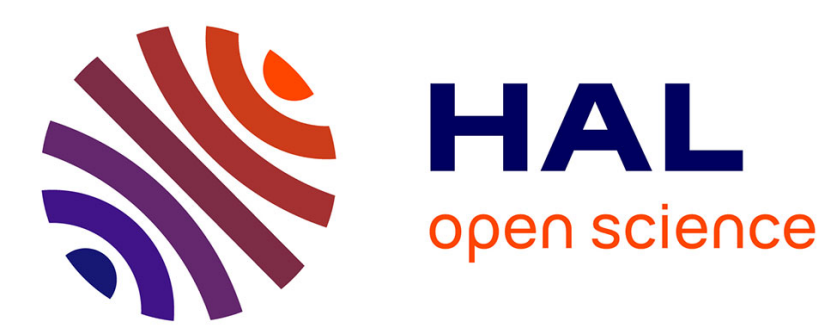

\title{
The zeroes of the partition function of the random energy model
}

Bernard Derrida

\section{To cite this version:}

Bernard Derrida. The zeroes of the partition function of the random energy model. Physica A : Statistical Mechanics and its Applications, 1991, 177 (1), pp.31-37. 10.1016/0378-4371(91)90130-5 . hal-03285599

\section{HAL Id: hal-03285599 \\ https://hal.science/hal-03285599}

Submitted on 21 Jul 2021

HAL is a multi-disciplinary open access archive for the deposit and dissemination of scientific research documents, whether they are published or not. The documents may come from teaching and research institutions in France or abroad, or from public or private research centers.
L'archive ouverte pluridisciplinaire HAL, est destinée au dépôt et à la diffusion de documents scientifiques de niveau recherche, publiés ou non, émanant des établissements d'enseignement et de recherche français ou étrangers, des laboratoires publics ou privés. 


\title{
The zeroes of the partition function of the random energy model
}

\author{
B. Derrida \\ Service de Physique Théorique de Saclay ', F-91191 Gif-sur-Yvette Cedex, France
}

\begin{abstract}
The random energy model is one of the simplest disordered models containing some of the physics of spin glasses. The zeroes of the partition function in the complex temperature plane are determincd for this modcl. They cither lie on simple lines or are dense in some regions of the complex plane.
\end{abstract}

Locating the zeroes of partition functions in the complex plane of the physical variables (magnetic field, temperature) has been a subject of constant interest in the theory of phase transitions for the last decades [1-9]. Phase transitions are expected to show up as accumulation points on the real axis of these complex zeroes. In the case of ferromagnetic interactions, the Lee and Yang theorem [1,2] guarantees that the zeroes in the complex plane of the magnetic field are all located along the imaginary axis. By contrast, in the complex plane of temperature, the patterns of zeroes can have more complicated structures [3]: isolated points, lines, areas and even fractals [10].

Several attempts of understanding disordered systems by looking at the distribution of zeroes in the complex plane have been tried [11,12]. Except for establishing the existence of Griffiths [13] singularities in diluted ferromagnets, where the proof is based on an estimation of the density of zeroes in the complex plane of the magnetic field, little progress has been made so far in disordered systems by looking at the complex zeroes of the partition function. Analyzing the patterns of zeroes calculated numerically is usually very hard because of the system sizes (the number of zeroes is very small) and of the sample to sample fluctuations, which are usually very large for small systems.

Recently, the zeroes of the partition function [14] of the random energy [15] model (which is one of the simplest models containing some of the physics of spin glasses) have been studied numerically, and they were found to have rather simple structures: lines and areas. The goal of the present paper is to describe these zeroes by an analytical approach. The main part of the argument is a direct generalization of an idea presented recently in the case of directed paths [16]. The main result is shown in fig. 1 .

The random energy model (REM) was introduced as a very simplified spin glass

'Laboratoire de la Direction des Sciences de la Matière du Commissariat à l'Energie Atomique. 
model $[15,17]$ which consists of $2^{N}$ energy levels randomly distributed according to a Gaussian distribution $P(E)$,

$$
P(E)=\frac{1}{\sqrt{\pi N}} \exp \left(-\frac{E^{2}}{N}\right) .
$$

The main reason the REM is soluble is that the energies of the $2^{N}$ configurations are independent. So a given sample is characterized by $2^{N}$ numbers $E_{a}$ randomly chosen according to (1) and the partition function corresponding to this sample is, as usual in statistical mechanics,

$$
Z=\sum_{a=1}^{2^{N}} \exp \left(-\frac{E_{a}}{T}\right)
$$

The goal, now, is to locate the zeroes of $Z$ in the complex plane of the temperature $T$. To do so, it is useful to obtain first the typical behaviour of the modulus $|Z|$ of $Z$ for any complex temperature $T$. This can be done by using the same approach as in ref. [16].

If we define.$(E)$ as the number of configurations in the range of energy $E, E+\Delta E$, i.e.

$$
\mathscr{f}(E)=\sum_{a} y_{a}
$$

where $y_{a}=1$ if $E \leqslant E_{a} \leqslant E+\Delta E$ and $y_{a}=0$ otherwise ( $\Delta E$ is a small interval $\Delta E \sim N^{-x}$ with for example $x=\mathscr{C}(1))$, then the partition function $Z$ is given by

$$
Z=\sum_{E} f(E) \exp \left(-\frac{E}{T}\right) \text {. }
$$

The number $\mathscr{N}(E)$ is sample dependent, and from (1) and (3) one knows its average:

$$
\langle\mathscr{N}(E)\rangle=2^{N} \frac{1}{\sqrt{\pi N}} \exp \left(-\frac{E^{2}}{N}\right) \Delta E .
$$

Because the $2^{N}$ energies $E_{a}$ are statistically independent, the variables $y_{a}$ in (3) are also independent. This allows one to calculate the fluctuations of $f(E)$ :

$$
\begin{aligned}
\left\langle\mathscr{V}^{2}(E)\right\rangle-\langle\mathscr{V}(E)\rangle^{2} & =\sum_{a}\left\langle y_{a}\right\rangle-\left\langle y_{a}\right\rangle^{2} \\
& =2^{N}\left\{P(E) \Delta E-[P(E) \Delta E]^{2}\right\} \simeq\langle\mathscr{V}(E)\rangle .
\end{aligned}
$$

By looking at (5) and (6), one can show that there are two different ranges of energies $E$. Defining $\epsilon_{\mathrm{c}}$ by

$$
\epsilon_{\mathrm{c}}=\sqrt{\log 2} \text {, }
$$

one sees that if $|E| / N\left\langle\epsilon_{\mathrm{c}}\right.$, then $\langle\mathscr{N}(E)\rangle \gg 1$ and the fluctuations of $\mathscr{f}(E)$ are small 
compared to its average. In that range one expects the typical number $\mathcal{N}_{\text {typ }}(E)$ to be of the form

$$
\mathscr{N}_{\text {typ }}(E)=\langle\mathscr{N}(E)\rangle+\eta(E)\langle\mathscr{N}(E)\rangle^{1 / 2},
$$

where $\eta(E)$ is a random number of order 1 (when $N$ is large) which fluctuates in sign and in amplitude from one energy interval $E, E+\Delta E$ to the next.

In the other range of energies defined by $|E| / N>\epsilon_{\mathrm{c}}$, the average number of configurations $\langle\mathscr{N}(E)\rangle$ is much smaller than 1 . Therefore, the probability of finding at least one configuration at these energies is exponentially small for large $N$, implying that

$$
\mathscr{N}_{\text {typ }}(E)=0 \text {. }
$$

From the above argument, we see that the computation of the partition function $Z_{\text {typ }}$ at any complex temperature $T$ in the typical case is reduced in the large $N$ limit to calculating

$$
Z_{\mathrm{lyp}}=A+B
$$

with

$$
A=\sum_{|E| / N<\epsilon_{\mathrm{c}}}\langle\mathcal{N}(E)\rangle \exp \left(-\frac{E}{T}\right)
$$

and

$$
B=\sum_{|E| / N<\epsilon_{\mathrm{c}}} \eta(E)\langle\mathscr{N}(E)\rangle^{1 / 2} \exp \left(-\frac{E}{T}\right) .
$$

As in ref. [16], by cstimating $A$ and $B$ for large $N$, we will sce that there exist three different phases.

The sum $A$ can be calculated by a saddle point method. For a complex value of the temperature $T$, the saddle point is located at an energy $E / N=-(2 T)^{-1}$. Therefore the only question in estimating the sum $A$ is to know whether or not, when one integrates over $E$ from $-N \epsilon_{\mathrm{c}}$ to $N \epsilon_{\mathrm{c}}$, the integral contour can be deformed to pass through the saddle point $(-2 T)^{-1}$ and if the endpoints $\left( \pm N \epsilon_{\mathrm{c}}\right)$ of the integral are lower than this saddle point. It is easy to check that this is only the case when

$$
-\epsilon_{\mathrm{c}}+\left|\operatorname{Im}\left(\frac{1}{2 T}\right)\right|<\operatorname{Re}\left(\frac{1}{2 T}\right)<\epsilon_{\mathrm{c}}-\left|\operatorname{Im}\left(\frac{1}{2 T}\right)\right| .
$$

Therefore, when (13) is satisfied, the sum $A$ becomes

$$
A=\frac{1}{\sqrt{\pi N}} \int 2^{N} \exp \left(-\frac{E^{2}}{N}\right) \exp \left(-\frac{E}{T}\right) \mathrm{d} E=2^{N} \exp \left(\frac{N}{4 T^{2}}\right) .
$$

On the contrary, when (13) is not satisfied, $A$ is dominated by either the upper or the lower limit of the sum (11) and the result is 


$$
|A| \simeq \exp \left[N \epsilon_{\mathrm{c}}\left|\operatorname{Re}\left(\frac{1}{T}\right)\right|\right] .
$$

One should notice that in (15), one cannot predict the phase of $A$ because the encrgies which dominate the sum $A$ are the ground state and the lowest excited states. These energies have sample to sample fluctuations of order 1 [15], implying that the phase of $Z$ fluctuates.

The term $B$ given by (12) can also be calculated for large $N$. Because $\eta(E)$ is a random function of $E$, the integrand is no longer an analytic function of $E$ and thus there is no way of calculating the sum (12) by deforming a contour in the complex plane of $E$. On the other hand, because the values of $\eta(E)$ at different energies $E$ are essentially uncorrelated, the term of largest modulus in the sum (12) dominates the amplitude of $B$ (the randomness of $\eta(E)$ prevents this largest term from being completely cancelled by the terms coming from the other energies). Therefore

$$
|B| \simeq \max _{-\epsilon_{\mathrm{q}} \leqslant E / N \leqslant \mathrm{\epsilon}_{\mathrm{c}}}\langle. \mid(E)\rangle^{1 / 2}\left|\exp \left(-\frac{E}{T}\right)\right| .
$$

As in the case of the sum $A$, there are two possible ranges of temperature.

In the first range of temperature defined by

$$
-\epsilon_{c} \leqslant \operatorname{Re}\left(-\frac{1}{T}\right) \leqslant \epsilon_{c},
$$

the energy $E$ which dominates the sum $B$ is $E / N=-\operatorname{Re}(1 / T)$, and the sum $B$ is given by

$$
|B| \simeq 2^{N / 2} \exp \left\{\frac{N}{2}\left[\operatorname{Re}\left(\frac{1}{T}\right)\right]^{2}\right\}
$$

whereas when (17) is not satisfied, the sum (12) is dominated by the ground state energy $E / N= \pm \epsilon_{\mathrm{c}}$ and one finds that

$$
|B| \simeq \exp \left[\frac{N \epsilon_{\mathrm{c}}}{2} \operatorname{Re}\left(\frac{1}{T}\right)\right] .
$$

Having obtained the large $N$ behaviours (14), (15), (18) and (19) of $A$ and $B$ under conditions (13) and (17), we can use (10) to obtain the modulus of $Z$ at any temperature. One finds three phases I, II and II as shown in fig. Ia, with

$$
\begin{array}{ll}
\text { in phase I } & \frac{\log |Z|}{N}=\log 2+\operatorname{Re}\left(\frac{1}{4 T^{2}}\right), \\
\text { in phase II } & \frac{\log |Z|}{N}=\epsilon_{\mathrm{c}}\left|\operatorname{Re}\left(\frac{1}{T}\right)\right|,
\end{array}
$$


(a)

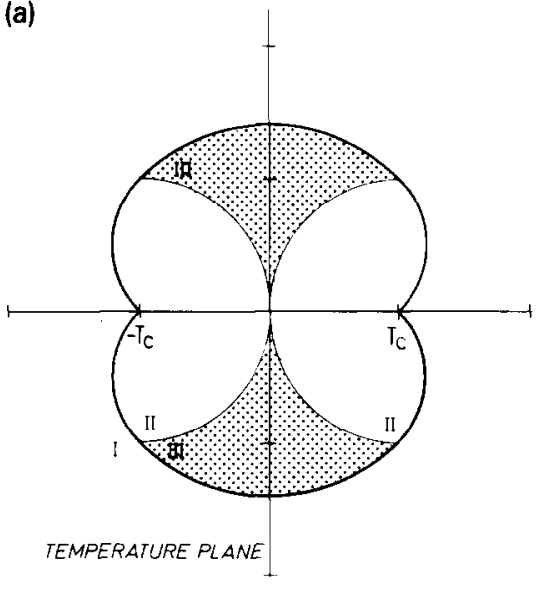

(b)

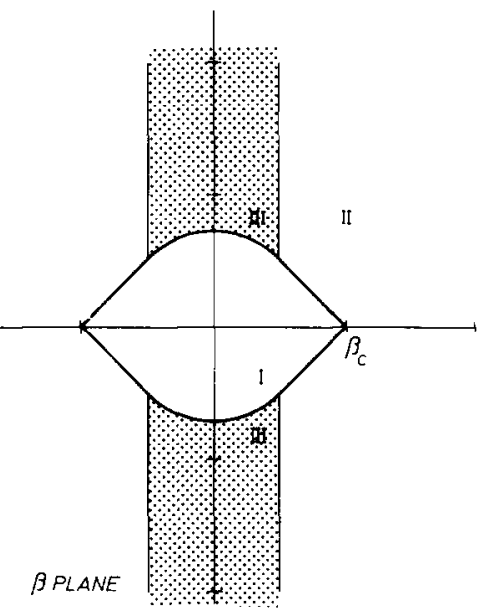

Fig. 1. The complex plane of temperature (a) or of $\beta$ (b). The zeroes are dense in the region III. The boundaries I-II and I-III are also lines of zeroes. The density in the $\beta$ plane is given in (28).

in phase III $\frac{\log |Z|}{N}=\frac{\log 2}{2}+\frac{1}{2}\left[\operatorname{Re}\left(\frac{1}{T}\right)\right]^{2}$.

A simpler way of looking at these three phases is to look at the complex plane (of $\left.\beta=\beta_{1}+\mathrm{i} \beta_{2}=1 / T\right)$. In the $\beta$ plane, the three phases are shown in fig. $1 \mathrm{~b}$ and the corresponding expressions of $\log |Z| / N$ are given by

phase I $\quad \frac{\log |Z|}{N}=\frac{1}{4} \beta_{\mathrm{c}}^{2}+\frac{1}{4}\left(\beta_{1}^{2}-\beta_{2}^{2}\right)$,

phase II $\frac{\log |Z|}{N}=\frac{1}{2} \beta_{\mathrm{c}}\left|\beta_{1}\right|$,

phase III $\quad \frac{\log |Z|}{N}=\frac{1}{8} \beta_{\mathrm{c}}^{2}+\frac{1}{2} \beta_{1}^{2}$,

where $\beta_{c}=2 \sqrt{\log 2}$ and the boundaries between these phases in the first quadrant are given by

phase I, phase II: $\beta_{1}+\beta_{2}=\beta_{\mathrm{c}}, \frac{1}{2} \beta_{\mathrm{c}}<\beta_{1}<\beta_{\mathrm{c}}$,

phase II, phase III: $\beta_{1}=\frac{1}{2} \beta_{\mathrm{c}}, \beta_{2}>\frac{1}{2} \beta_{\mathrm{c}}$,

phase I, phase III: $\beta_{1}^{2}+\beta_{2}^{2}=\frac{1}{2} \beta_{\mathrm{c}}^{2}, \beta_{1}<\frac{1}{2} \beta_{\mathrm{c}}$.

The boundaries in the other quadrants follow by symmetry.

Once the expression of $\log |Z| / N$ is known, the density of zeroes $\rho\left(\beta_{1}, \beta_{2}\right)$ in the complex plane of $\beta$ can be easily obtained through the following expression:

$$
\rho\left(\beta_{1}, \beta_{2}\right)=\frac{1}{2 \pi}\left(\frac{\mathrm{d}^{2}}{\mathrm{~d} \beta_{1}^{2}}+\frac{\mathrm{d}^{2}}{\mathrm{~d} \beta_{2}^{2}}\right)\left(\frac{\log |Z|}{N}\right) .
$$


This expression can be understood by considering the following example: take a polynomial $f(\beta)$ with $P$ complex zeroes $\gamma_{1}, \ldots, \gamma_{P}$,

$$
f(\beta)=\prod_{i=1}^{p}\left(\beta-\gamma_{i}\right) \text {. }
$$

Clearly one has

$$
\log (|f(\beta)|)=\sum_{i=1}^{P} \log \left|\beta-\gamma_{i}\right| .
$$

and using the fact (which can be checked for example by calculating the Laplacian of $\log \left(\beta_{1}^{2}+\beta_{2}^{2}+\eta^{2}\right)$ and taking then the limit $\left.\eta \rightarrow 0\right)$ that

$$
\left(\frac{\mathrm{d}^{2}}{\mathrm{~d} \beta_{1}^{2}}+\frac{\mathrm{d}^{2}}{\mathrm{~d} \beta_{2}^{2}}\right) \log \left(\beta_{1}^{2}+\beta_{2}^{2}\right)=4 \pi \delta\left(\beta_{1}\right) \delta\left(\beta_{2}\right)
$$

one obtains

$$
\left(\frac{\mathrm{d}^{2}}{\mathrm{~d} \beta_{1}^{2}}+\frac{\mathrm{d}^{2}}{\mathrm{~d} \beta_{2}^{2}}\right) \log (|f(\beta)|)=2 \pi \sum_{i=1}^{r} \delta\left(\beta_{1}-\gamma_{i}^{(1)}\right) \delta\left(\beta_{2}-\gamma_{i}^{(2)}\right) .
$$

where $\gamma_{i}^{(1)}$ and $\gamma_{i}^{(2)}$ are the real and imaginary parts of the zero $\gamma_{i}$. So we see that (22) gives the right density for the polynomial (23).

From (21) and (22), we see that there are no zeroes (or at least the density of zeroes vanishes) in phases I and II and that the density of zeroes is uniform in phase III as shown in fig. $1 \mathrm{~b}$ (complex plane of $\beta$ ).

$$
\rho\left(\beta_{1}, \beta_{2}\right)=\frac{1}{2 \pi} \text {. }
$$

This leads to a region III in fig. Ia (complex plane of temperature) with a non-constant density of zeroes which can be easily obtained from (27) by using the transformation $T=1 / \beta$.

In addition to the uniform density (27) of zeroes in region III, one can also obtain from (21) and (22) the densities of zerocs along the lines which separate the different phases of fig. $1 \mathrm{~b}$. The total density $\rho\left(\beta_{1}, \beta_{2}\right)$ which results from $(21)$ and $(22)$ is, in the first quadrant,

$$
\begin{aligned}
\rho\left(\beta_{1}, \beta_{2}\right)= & \frac{1}{2 \pi} \theta\left(\frac{1}{2} \beta_{\mathrm{c}}-\beta_{1}\right) \theta\left(\beta_{1}^{2}+\beta_{2}^{2}-\frac{1}{2} \beta_{\mathrm{c}}^{2}\right) \\
& +\frac{1}{2 \pi}\left(\beta_{\mathrm{c}}-\beta_{1}\right) \delta\left(\beta_{1}+\beta_{2}-\beta_{\mathrm{c}}\right) \theta\left(\beta_{1}-\frac{1}{2} \beta_{\mathrm{c}}\right) \\
& +\frac{1}{2 \pi} \frac{1}{2} \beta_{\mathrm{c}}^{2} \delta\left(\beta_{1}^{2}+\beta_{2}^{2}-\frac{1}{2} \beta_{\mathrm{c}}^{2}\right) \theta\left(\frac{1}{2} \beta_{\mathrm{c}}-\beta_{1}\right) .
\end{aligned}
$$

The distribution in the other quadrants follows by symmetry. The density seems to be 
in good agreement with the numerical results of Moukarzel and Parga [14]. It is noteworthy that the boundaries between phases I and II and between phases I and III are lines of zeroes whereas the separation between phases II and III is not. One can notice that a line of zeroes crosses the real axis at $T_{\mathrm{c}}$ with an angle $\frac{1}{4} \pi$ as expected [18] for a system with a discontinuous specific heat [15].

One should be able to extend the above calculation to distributions of energies other than (1) (for example binomial distributions).

\section{References}

[1] C.N. Yang and T.D. Lee, Phys. Rev. 87 (1952) 404.

[2] T.D. Lee and C.N. Yang, Phys. Rev. 87 (1952) 410.

[3] M.E. Fisher, in: Lectures in Theoretical Physics, vol. 7 (University of Colorado, Boulder, 1965).

[4] R.A. Abe and S. Katsura, Prog. Theor. Phys. 43 (1970) 1402.

[5] D. Ruelle, Commun. Math. Phys. 31 (1973) 265.

[6] R.B. Pearson, Phys. Rev. B 26 (1982) 6285.

[7] W. van Saarloos and D.A. Kurtze, J. Phys. A 17 (1984) 1301.

[8] D.W. Wood, J. Phys. A 18 (1985) L481.

[9] J. Stephenson, J. Phys. A 20 (1987) 4513.

[10] B. Derrida, L. De Sèze and C. Itzykson, J. Stat. Phys. 33 (1983) 559.

[11] R. Rammal, Thèse à l'Université de Grenoble (1981).

[12] Y. Ozeki, H. Nishimori, J. Phys. Soc. Jpn. 57 (1988) 1087.

[13] R.B. Griffiths, Phys. Rev. Lett. 23 (1969) 17.

[14] C.F. Moukarzel and N. Parga, preprint (1991).

[15] B. Derrida, Phys. Rev. B 24 (1981) 2613.

[16] J. Cook and B. Derrida, J. Stat. Phys. 61 (1990) 961.

[17] E. Gardner and B. Derrida, J. Phys. A 22 (1989) 1975.

[18] C. Itzykson, R.B. Pearson and J.B. Zuber, Nucl. Phys. B 220 (1983) 415. 